\title{
Review Article \\ PRP Augmentation for ACL Reconstruction
}

\author{
Luca Andriolo, ${ }^{1}$ Berardo Di Matteo, ${ }^{1}$ Elizaveta Kon, ${ }^{2}$ Giuseppe Filardo, ${ }^{1}$ \\ Giulia Venieri, ${ }^{1}$ and Maurilio Marcacci ${ }^{1}$ \\ ${ }^{1}$ II Orthopaedic and Traumatology Clinic, Biomechanics and Technology Innovation Laboratory, \\ Rizzoli Orthopaedic Institute, Via di Barbiano No. 1/10, 40136 Bologna, Italy \\ ${ }^{2}$ Nano-Biotechnology Laboratory, Rizzoli Orthopaedic Institute, Via di Barbiano No. 1/10, 40136 Bologna, Italy
}

Correspondence should be addressed to Luca Andriolo; lucas.andriolo@gmail.com

Received 29 May 2014; Accepted 15 August 2014

Academic Editor: Tomokazu Yoshioka

Copyright (C) 2015 Luca Andriolo et al. This is an open access article distributed under the Creative Commons Attribution License, which permits unrestricted use, distribution, and reproduction in any medium, provided the original work is properly cited.

\begin{abstract}
Current research is investigating new methods to enhance tissue healing to speed up recovery time and decrease the risk of failure in Anterior Cruciate Ligament (ACL) reconstructive surgery. Biological augmentation is one of the most exploited strategies, in particular the application of Platelet Rich Plasma (PRP). Aim of the present paper is to systematically review all the preclinical and clinical papers dealing with the application of PRP as a biological enhancer during ACL reconstructive surgery. Thirty-two studies were included in the present review. The analysis of the preclinical evidence revealed that PRP was able to improve the healing potential of the tendinous graft both in terms of histological and biomechanical performance. Looking at the available clinical evidence, results were not univocal. PRP administration proved to be a safe procedure and there were some evidences that it could favor the donor site healing in case of ACL reconstruction with patellar tendon graft and positively contribute to graft maturation over time, whereas the majority of the papers did not show beneficial effects in terms of bony tunnels/graft area integration. Furthermore, PRP augmentation did not provide superior functional results at short term evaluation.
\end{abstract}

\section{Introduction}

Anterior cruciate ligament (ACL) tears are among the most common sport-related injuries and therefore ACL reconstructive surgery is one the most frequently performed procedures in the field of sport medicine [1]. Epidemiological data reveal that the majority of patients are young and sportactive, with high expectations in terms of functional recovery and return to sport [2]. There is a flourishing literature concerning ACL reconstructive procedures: several different techniques have been documented over the years and, despite overall good clinical outcomes reported at mid/long-term followup, ACL is still on the edge of current clinical and preclinical research [3].

ACL surgery (and the related research) is a classic example of integration between biomechanics and biology: the progress made in recent decades can be attributed to the big steps forward in the knowledge of both the mechanical and biological properties of ACL and its healing process [48]. Although almost all available techniques can provide satisfactory results, ACL reconstruction is not a " $100 \%$-success" procedure: not all patients are able to regain their previous sport activity level and many factors could influence the clinical outcome, such as the type of graft used and the preop. knee laxity $[2,9]$. This is a major concern since ACL injured patients can be a very demanding category, especially professional sport players who also need to return to the playing field as soon as possible. Current research is therefore investigating novel strategies to enhance ACL healing, to reduce the failure rate and accelerate recovery time. Among the different available options, biological augmentation is the most sought after approach and recently platelet rich plasma (PRP), which is an autologous blood derivative, largely applied in orthopaedic practice especially in the treatment of degenerative cartilage and tendon lesions, is gaining increasing interest [10-12]. PRP is a source of several growth factors (GFs) and other bioactive molecules that might promote tissue healing and regulate joint homeostasis $[13,14]$. It is an easily available product obtained directly from the venous blood of the patient and its use is allowed also in athletes, since antidoping regulations do not consider this blood derivative as a banned substance [15]. Furthermore, it is 
a versatile product since it can be prepared and used directly in the operating theatre, through intra-articular injections or in the shape of a membrane that can be placed directly onto the target site. The healing potential of PRP has been shown in several preclinical and clinical studies $[13,16,17]$, revealing that its action is directed toward all the articular tissues, ranging from meniscus to cartilage and even soft tissues like synovium, tendons, and ligaments: the effects of PRP are several, including an anabolic stimulus toward cells, an increase in extracellular matrix deposition, reduction of proapoptotic signals, and even an anti-inflammatory effect in the joint environment [13].

In light of such potential, the possibility of applying this biological product to enhance ACL reconstructive surgery appears attractive: PRP not only might promote a better and faster ligamentization of the graft used for ACL reconstruction and reduce the proinflammatory factors released immediately after surgery, but might also contribute to a better integration of the graft within the bone tunnels, thus avoiding their enlargement and failure over time. Furthermore, PRP could be used to accelerate healing and reduce donor-site morbidity at the harvest site of the tendon graft.

The aim of the present paper is to review systematically the current preclinical and clinical evidence concerning the application of PRP as a biological augmentation, to determine safety and efficacy of this biological approach to improve ACL reconstruction surgery.

\section{Materials and Methods}

A systematic review of the literature was performed on the use of PRP in ACL reconstruction. The search was conducted on the PubMed database on February 20th, 2014 using the following parameters: ((ACL) OR (ACL reconstruction) OR (ACL lesion)) AND ((PRP) OR (platelet rich plasma) OR (platelet gel) OR (platelet derived) OR (platelet concentrate)). The guidelines for Preferred Reporting Items for Systematic Reviews and Meta-Analysis (PRISMA) were used. Screening process and analysis were conducted separately by 2 independent observers (BDM and LA).

First, the articles were screened by title and abstract. The following inclusion criteria for relevant articles were used during the initial screening of titles and abstracts: clinical and preclinical reports of any level of evidence, written in English language, with no time limitation, on the use of PRP in ACL reconstruction, and reporting results on PRP effects. Exclusion criteria were articles written in other languages, reviews, or studies analyzing other applications of PRP in knee surgery not related to ACL procedures. In the second step, the full texts of the selected articles were screened, with further exclusions according to the previously described criteria. Moreover, the articles not reporting clinical, MRI, or histologic results were excluded. Reference lists from the selected papers were also screened. A flowchart of the systematic review is provided in Figure 1. Relevant data were then extracted and collected in a unique database with the consensus of the two observers to be analyzed for the purposes of the present paper.

\section{Results}

The database search identified 60 records, and the abstracts were screened and selected according to the inclusion/exclusion criteria. As shown in Figure 1, a total of 33 fulltext articles were assessed for eligibility. Four articles [1821] did not fulfill the criteria and were further excluded, and 3 articles came from the screening of the reference lists, leading to a total of 32 studies included in the final analysis. A detailed description of preclinical studies is reported in Table 1, whereas clinical studies are summarized in Table 2 and discussed in more detail in the following paragraph.

3.1. Clinical Evidence. Fifteen clinical trials were selected according to the inclusion criteria: 11 were randomized controlled trials, 3 were prospective comparative studies, and 1 was a retrospective comparative trial (Table 2).

With regard to the graft used to perform ACL reconstruction, in 9 studies, authors used hamstring tendons (gracilis and semitendinosus), in 4 studies bone-patellar tendon-bone (BPB) graft, in 1 study allograft, and in 1 paper authors both hamstring tendons and BPB graft. Fourteen papers described a single-bundle reconstruction technique, whereas just in one trial a double-bundle procedure was used. Concerning PRP delivery methods, in 2 papers (both dealing with BPB graft) PRP was used to promote harvest site healing, in 1 paper PRP was applied intra-articularly by a suprapatellar injection at the very end of the surgical procedure, in 1 paper PRP was used just to coat the intra-articular portion of the graft, and in 11 papers it was administered both on the graft and in the bony tunnels to enhance the graft-bone integration. Among the delivery techniques used, in 2 studies peculiar approaches were applied. While most of the authors applied PRP on the graft surface, Sánchez et al. [22] placed PRP inside the graft through multiple intratendinous depots, while Radice et al. [23] used a more complex technique: before PRP application a bioabsorbable spongy membrane (Gelfoam, Pfizer, New York, NY) was carefully sutured on the harvested tendon and around the femoral bone plug in case of the BPB graft. Then, PRP was administered to allow the spongy membrane to absorb the PRP and avoid its dispersion in the articular space.

In all but one case PRP was activated before administration. Details regarding PRP preparation methods, number of patients included, patients' characteristics, and follow-up evaluations are included in Table 2.

To make the results more understandable, they will be discussed separately according to the specific aspects analyzed in the clinical trials:

(i) harvest site healing;

(ii) tendon graft maturation and bony tunnel/graft integration;

(iii) clinical results.

3.2. Healing of the Harvest Site. Two papers were specifically aimed at assessing the contribution of PRP in the healing of the harvest site after ACL reconstruction with $\mathrm{BPB}$ graft, and both of them reported positive outcomes with PRP. 


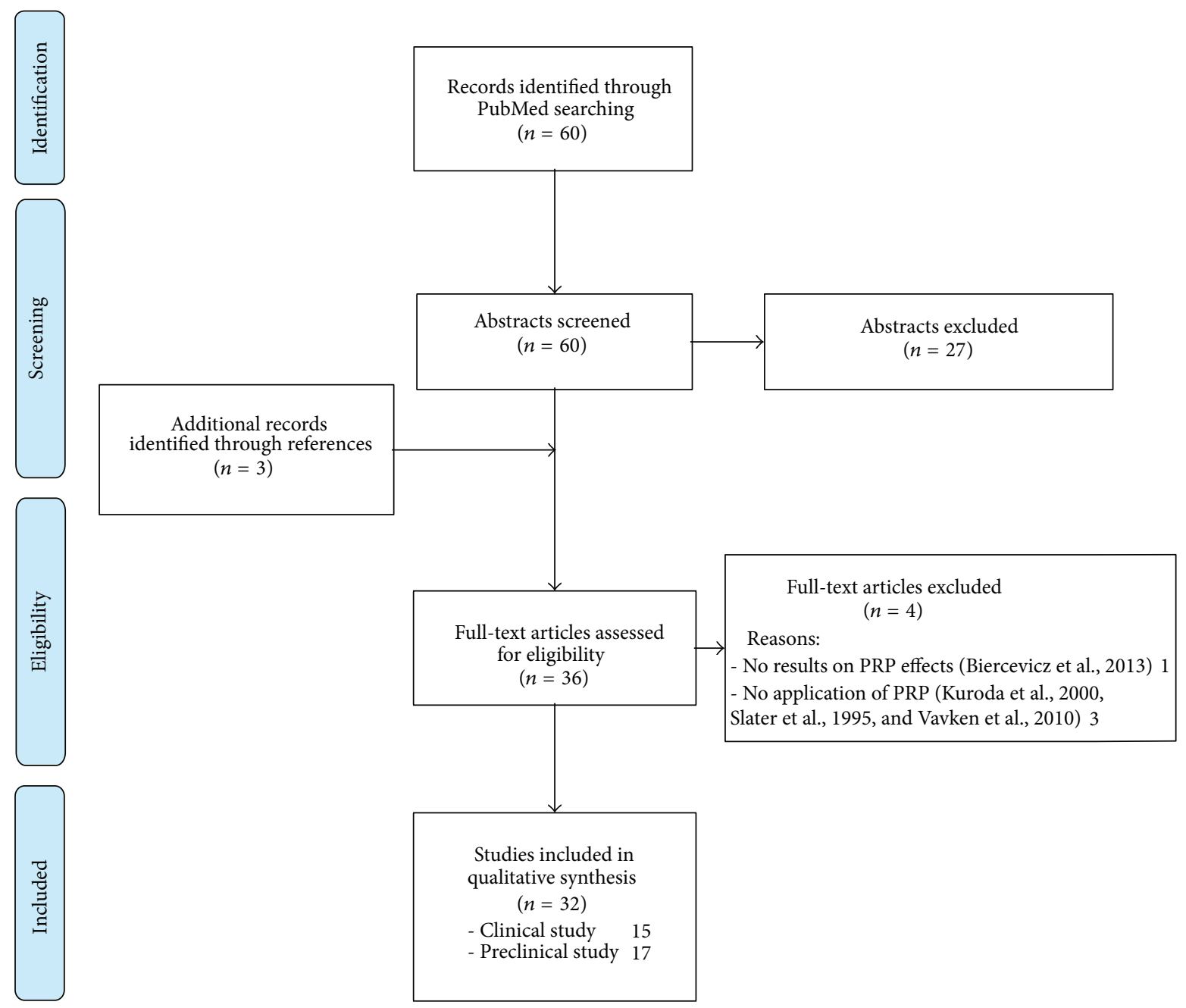

FIGURE 1: Flowchart of the systematic review.

In the study led by de Almeida et al. [24] two groups of patients (15 and 12, resp.) were randomized to receive or not receive PRP after BPB tendon harvesting. Total amount of $20-$ $40 \mathrm{~mL}$ of PRP was locally applied and then the peritendineum was sutured. Clinical and MRI evaluations were performed up to 6 months' after operation. The authors found that PRP augmentation determined a significantly smaller patellar tendon gap area with respect to the control group and also a lower postoperation pain reaction in the PRP group. However, at the final clinical evaluation no statistical intergroup difference was recorded in the questionnaires used or in the isokinetic test. In the paper authored by Cervellin et al. [25], 40 patients were included and divided into two treatment groups in the same manner. At 1-year followup, the PRP group showed statistically superior clinical outcomes when evaluated by the VISA-P score and, although not significant, a better bone healing when analyzed by MRI imaging, at both patellar and tibial defect sites (85\% of patients in PRP versus $60 \%$ in the control group).

3.3. Tendon Graft Maturation and Bony Tunnel/Graft Integration. Twelve papers examined outcome regarding (a) graft maturation over time and (b) graft integration in the bone tunnels.

Among the 6 studies reporting data about graft maturation, 4 of them were in favour of PRP augmentation, whereas 2 reported no intergroup difference. With respect to bony tunnels/graft area, 9 studies focused on the integration, documenting in 7 cases no advantage after PRP administration. The evolution over time was investigated more specifically in 3 trials focused on the bony tunnel widening: none demonstrated that PRP was able to prevent tunnels' enlargement over time.

Looking in more detail at the available literature, the first paper was published by Orrego et al. [53] in 2008: 108 patients were divided into 4 treatment groups due to the fact that the authors also used an autologous spongy bone graft (placed by interference fit in the femoral tunnel after graft fixation) as autologous augmentation. A control group was compared to PRP, bone plug, and PRP + bone plug groups. At 6 months MRI revealed a statistically significant advantage for the PRP group in terms of graft maturation (lower signal intensity at MRI) with respect to the control 


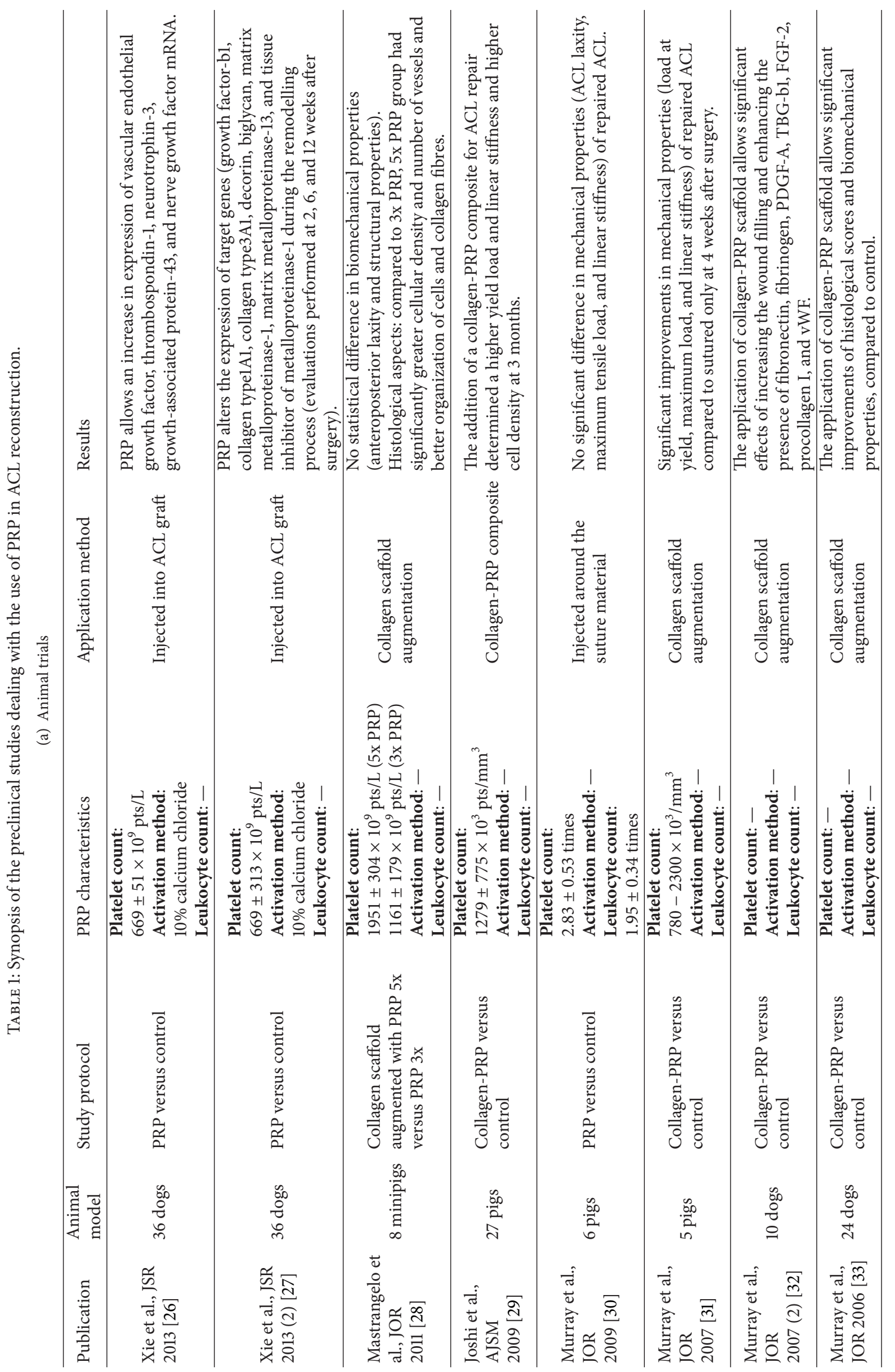




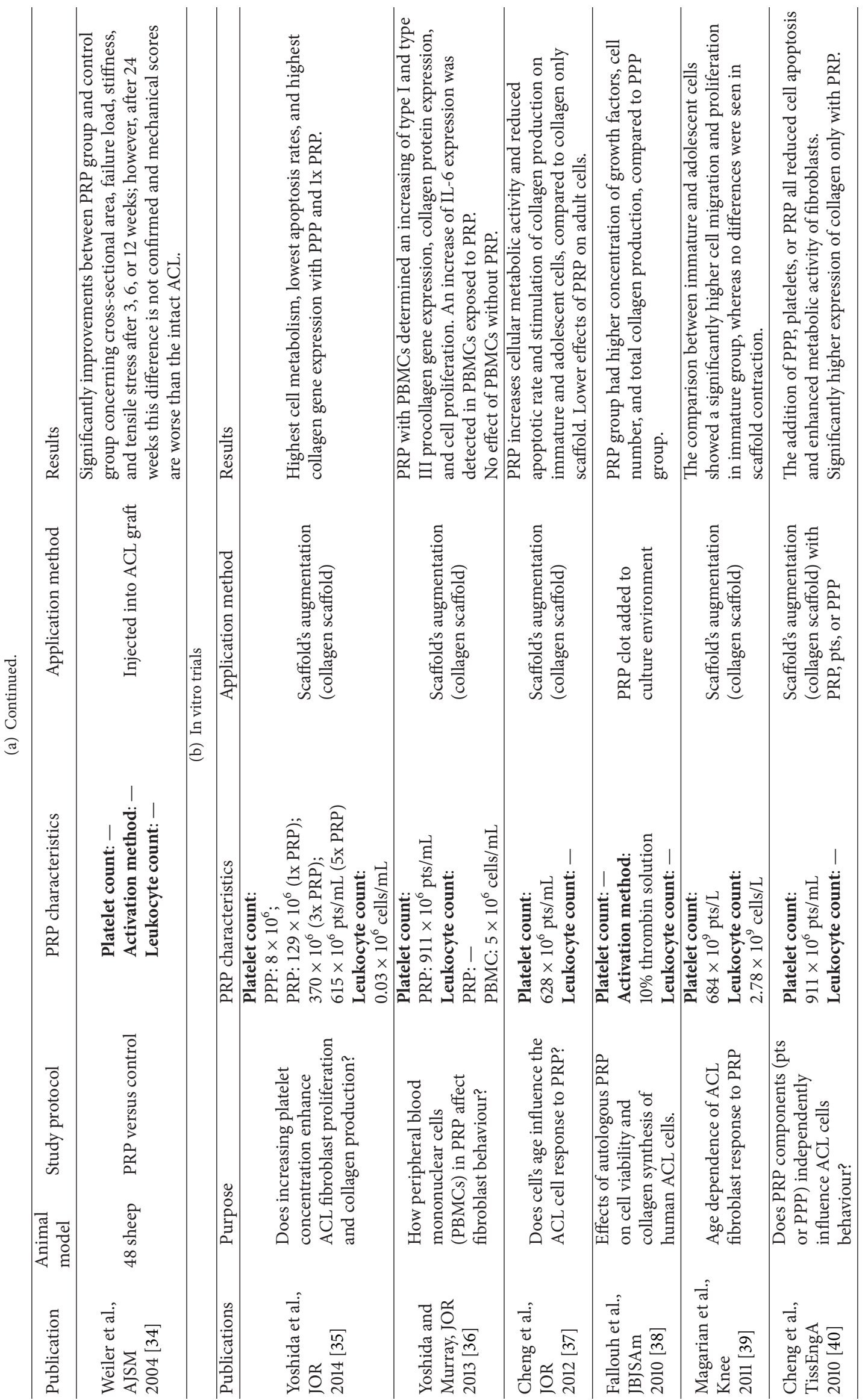




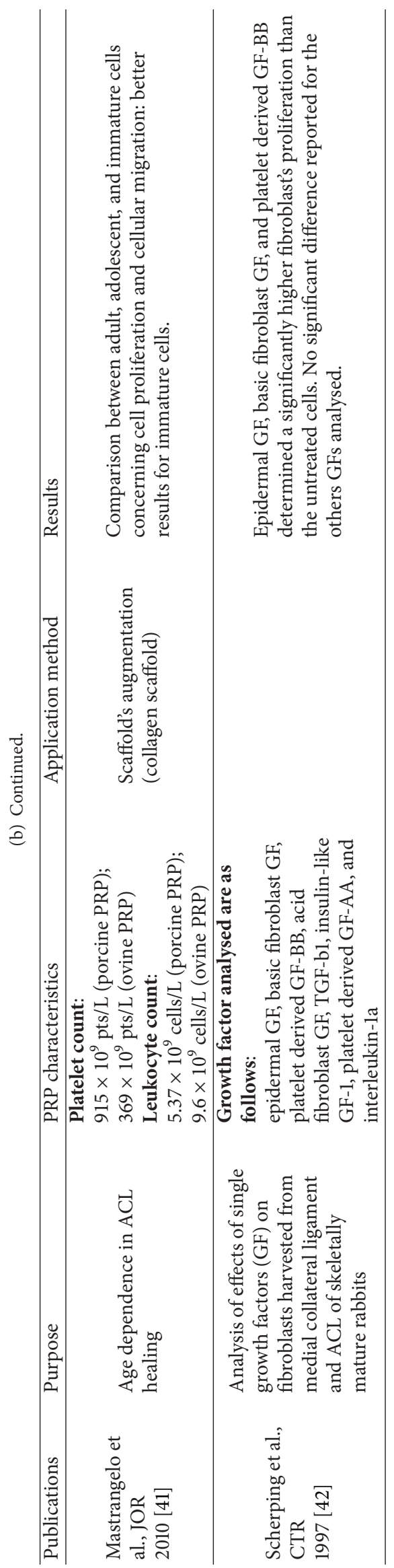




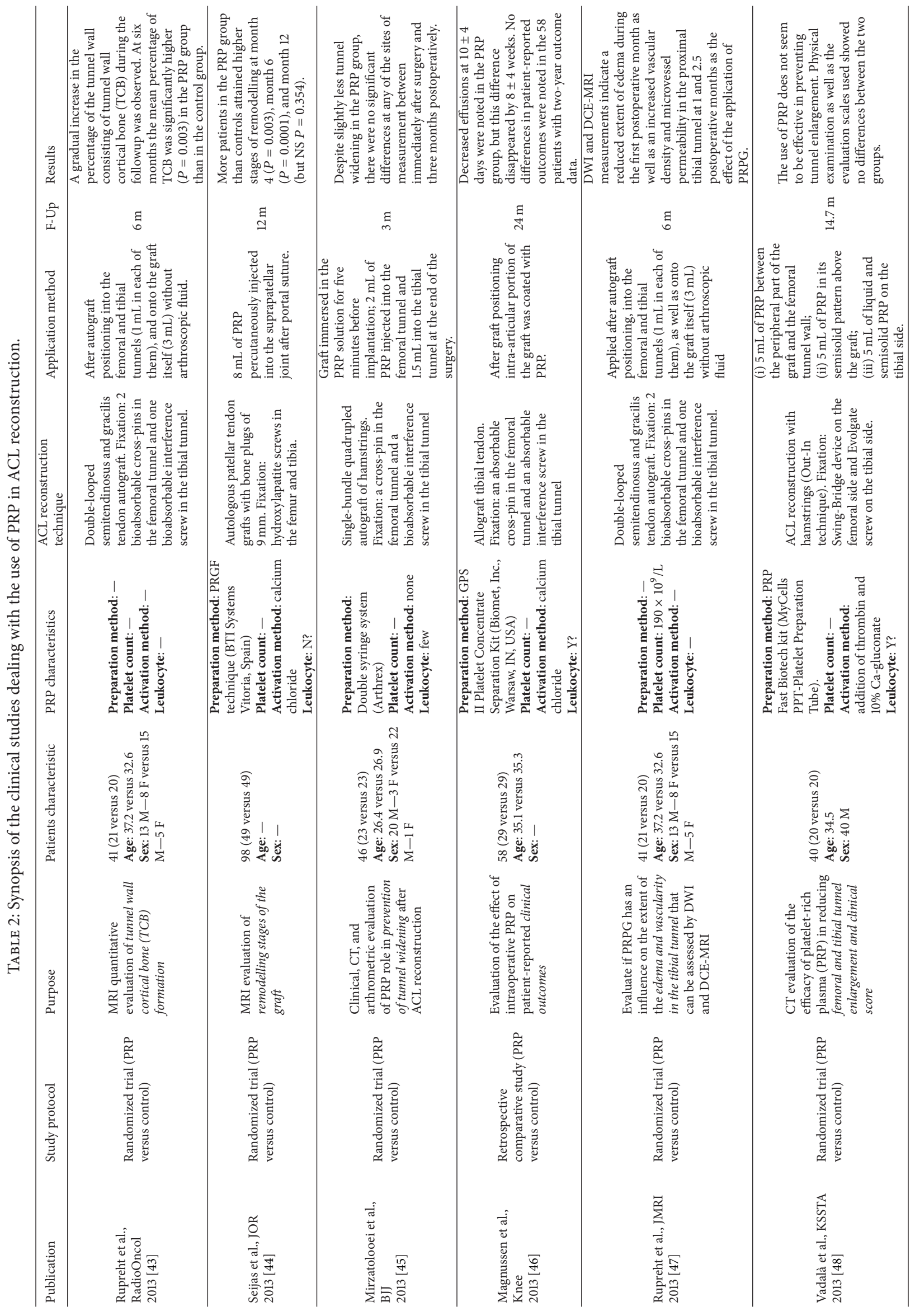




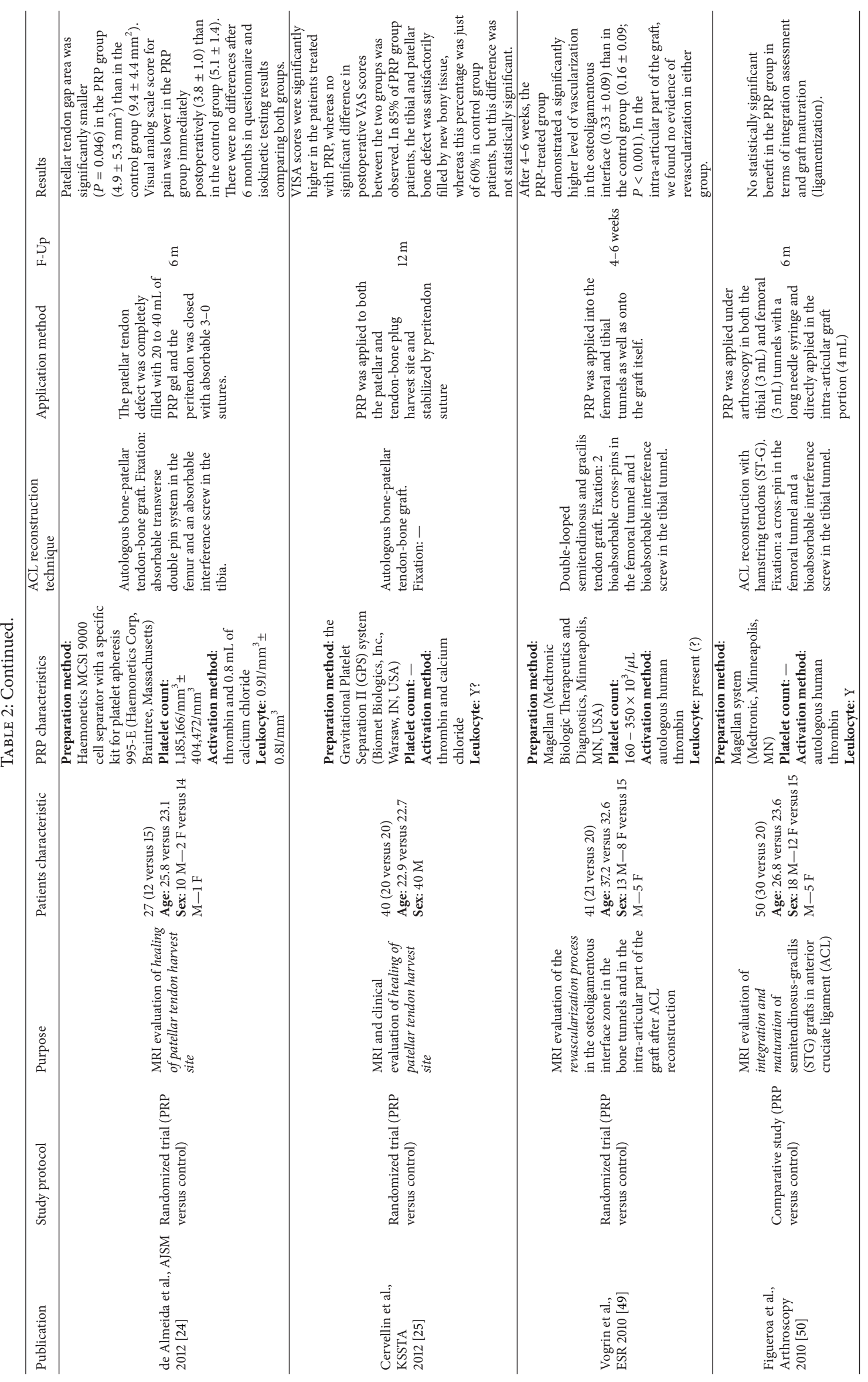




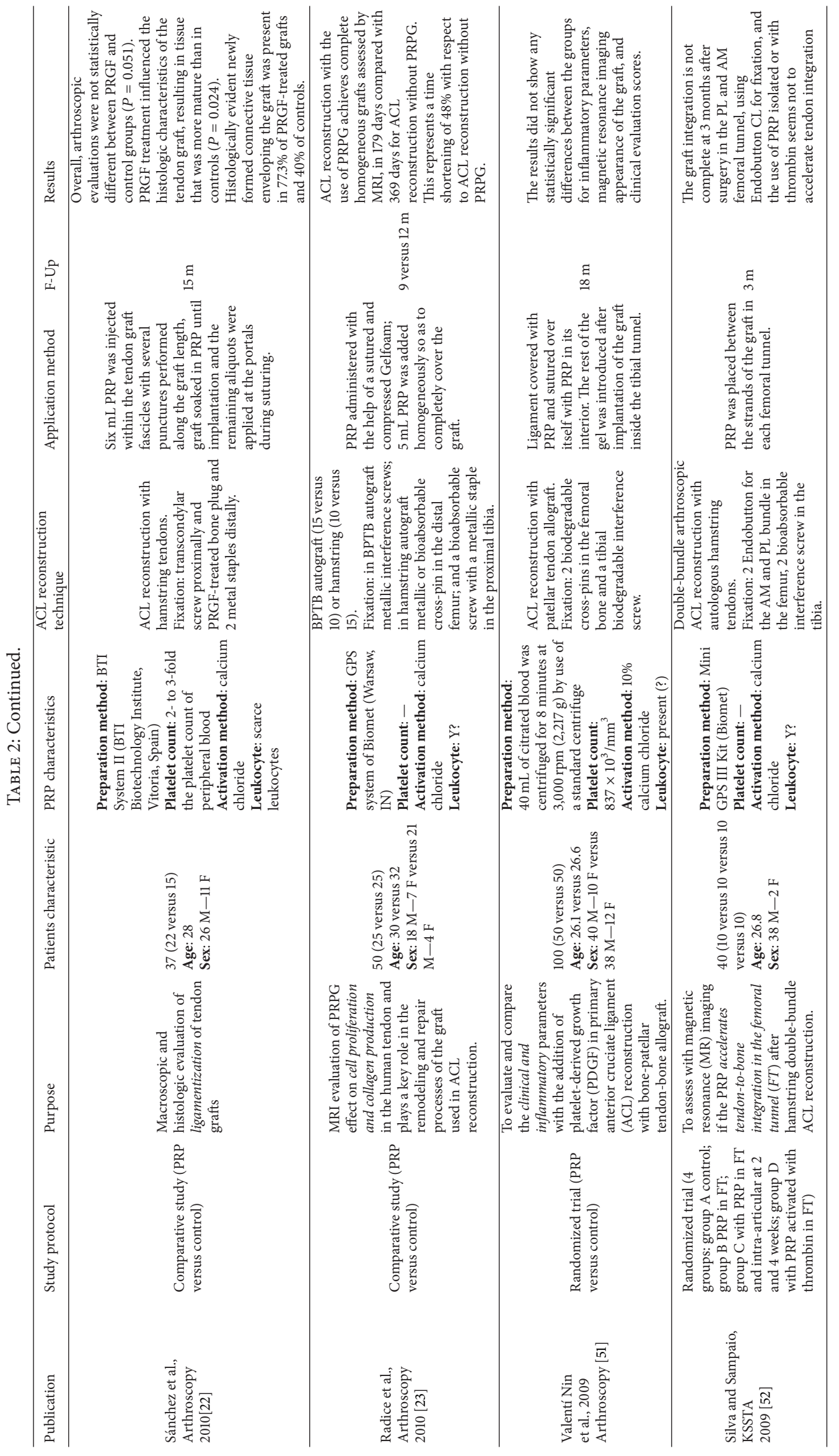




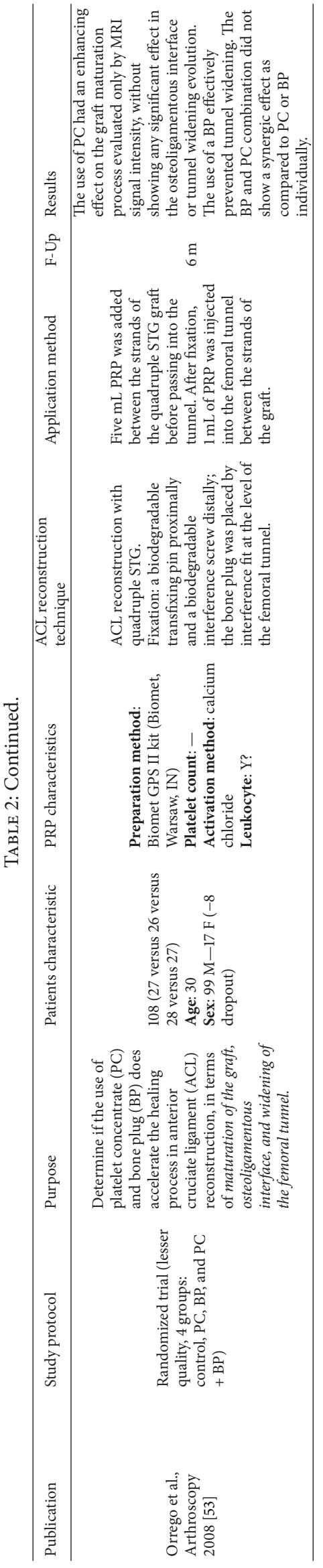


group. However, no differences were observed regarding the evaluation of osteoligamentous interface between the tunnels and the graft and, with regard to tunnel widening, the best results were achieved in the bone plug group which showed the lowest rate of widening, without further advantage from PRP administration. A positive influence of PRP local administration in graft maturation was also shown by other authors. The study authored by Radice et al. [23], who performed ACL reconstruction through both hamstring and PBP grafts, found a better maturation of the intra-articular portion of the graft in the PRP group. In particular, serial postoperation MRI assessments revealed that the mean time to achieve a homogenous "ligamentous-like" signal of the graft was 179 days in PRP group versus 362 days in control group. Subgroup analysis proved that the best responding category was the one where BPB graft reconstruction was performed. Similarly, Sánchez et al. [22] performed macroscopic and histologic evaluations in $37 \mathrm{ACL}$ patients (22 PRP and 15 control) who underwent second-look arthroscopies for other reasons. The authors found that, in patients who received PRP augmentation, there was a superior arthroscopic appearance of the graft (although not significant; $P=0.051$ ) and even biopsies revealed a superior tissue quality for PRP, with newly formed synovial-like tissue enveloping the graft in $77 \%$ of cases versus $40 \%$ of the control group. Seijas et al. [44] were the only authors that administered PRP by an intraarticular injection immediately after closing the arthroscopic portals, thus avoiding its selective delivery onto the graft or into the bony tunnels: the authors found that, despite this unselective administration, there was significant superior graft maturation in PRP group at MRI evaluation both at 3 and 6 months. Finally, with regard to positive PRP effects, Rupreht et al. were the only ones documenting benefit in the bony tunnel/graft area. In a randomized study [47] they showed that PRP administration reduced edema around the tibial tunnel during the first postoperation month and also increased vascular density and microvessel permeability in the proximal tibial tunnel at 1 and 2.5 months' MRI evaluation, thus suggesting that PRP is most effective in the early phases of healing. In another paper [43], the same authors reported that PRP increased cortical bone formation around the tibial tunnel wall at 6 months followup, thus suggesting that PRP might effectively contribute to graft-bone integration.

Among the studies documenting no beneficial effects of PRP administration, the first one was authored by Silva et al. [52] who evaluated the effect of PRP augmentation in promoting graft integration within the femoral tunnels. Forty patients were randomly divided into 4 groups: 10 were controls, 10 received PRP on the graft and in the femoral tunnels, 10 received the same treatment with thrombinactivated PRP, and 10 received further PRP intra-articular injections at 2 and 4 weeks after surgery. MRI controls 3 months after operation showed no statistical intergroup difference regarding maturation of fibrous interzone and, therefore, in osteoligamentous integration. Similar results at 6 months' evaluation were reported by Figueroa et al. [50] who treated 50 patients and analyzed MRI with the purpose of assessing graft maturation and osteoligamentous integration: no significant intergroup difference was found either in the first parameter or in the second. Another study by Vogrin et al. [49] investigated the vascularization at the interface between bone tunnels and graft, and along the intra-articular portion of the graft: a superior vascularization was found in the PRP group only at 4-6 weeks with MRI but not at 10-12 weeks, and no difference was observed with respect to the control group in the vascularization of the intraarticular part of the graft. Another randomized study by Nin et al. [51] on 100 patients revealed that PRP augmentation did not provide superior results in terms of clinical scores, biomechanical tests, and MRI parameters of graft maturation at 12 months evaluation. Furthermore, the authors found no difference either in postoperation swelling or in C-reactive protein levels (inflammatory index) at 10 days after operation Finally, with regard to the evolution of the bony tendon/graft area, beside the previously mentioned study by Orrego et el. [53], more recently Vadalà et al. in 2013 [48] analyzed the role of PRP in preventing bone tunnel enlargement after ACL reconstruction: CT evaluation in 40 patients at a mean of 14.7 months' followup did not reveal any beneficial effect of PRP and furthermore the clinical scores were not positively affected by this biological augmentation. In a similar randomized study on 46 patients led by Mirzatolooei et al. [45] the same findings were reported regarding bone tunnel widening, without any advantage using PRP.

3.4. Clinical Results. Seven studies reported clinical outcomes after ACL reconstructive surgery with or without PRP augmentation, focusing on the short-term outcome, with followup reported from 6 months to 2 years. None of them showed any statistical inter-group difference. Only Magnussen et al. in a comparative retrospective trial [46] observed a lower swelling reaction in the PRP group at a mean of 10 days after operation, but the difference was no longer significant after 8 weeks.

\section{Discussion}

The reduction of recovery time and prevention of reinjury after ACL reconstruction are the main goals of sport medicine surgeons. In recent years a number of studies have been published aimed at clarifying the best surgical techniques and considering the different types of grafts, the fixation devices, and other biomechanical and technical variables. Biological augmentation through PRP administration is one of the latest topics for researchers, with the aim of enhancing ACL reconstruction via the help of powerful biological agents. In vitro studies and also animal trials have highlighted overall encouraging results (Table 1), thus confirming the expectations about the potential of platelet-derived GFs in stimulating tissue healing: the use of PRP increased the expression of procollagen gene and collagen protein and also contributed to reduce apoptosis and stimulate fibroblast metabolic activity [35-42]. In the animal model it was also observed that PRP was able to determine superior biomechanical properties such as a higher tensile load and linear stiffness of the graft [26-34]. In light of these findings, the application 
of PRP augmentation in clinical practice appeared justified. However, this systematic review underlines that results are more controversial when looking at clinical published data.

According to the clinical trials currently available, it is possible to highlight some important findings. The first one regards the safety of this approach. The intraoperative use of PRP proved to be safe: in none of the clinical trials considered adverse events related specifically to its use could be identified, and no infections or other complications were reported after PRP administration. PRP actually proved to even reduce the surgical morbidity in two papers [24, 25] specifically investigating its role in promoting graft harvest site healing. In both cases a BPB graft was used for ACL reconstruction and PRP contributed to better healing response evaluated radiographically and, in one case [25], this difference was also reflected in the clinical score (VISA-P). These findings underline that PRP is beneficial in the tendon healing process and confirm the results obtained in other studies dealing with PRP treatment of patellar tendon disease [10]. Based on these results, PRP could be considered as a valid option to address the problem of donor-site morbidity when the patellar tendon is the surgeon's choice for graft harvesting.

More controversial are the results in terms of biological efficacy on the reconstructed ACL. The application of PRP has been hypothesized to improve graft integration within the tunnels. Concerning the osteoligamentous integration, Rupreht et al. showed increased vascular density and microvessel permeability in the proximal tibial tunnel at 1 and 2.5 months' MRI evaluation, as well as reduced bone edema around the tunnel and increased cortical bone formation around the tibial tunnel wall at 6 months followup [43, 47]. Vogrin et al. [49] also showed a significantly higher neovascularization 4-6 weeks after PRP administration, but the advantage of PRP was not confirmed at further followup, thus suggesting that PRP is most effective in the early phases of healing. Other studies did not show any difference in osteoligamentous integration $[50,52]$, and the three studies, all randomized trials $[45,48,53]$ focused on the evolution of bony tunnel/graft area, found that biological augmentation did not contribute to preventing tunnel enlargement with respect to the control group. Therefore, this particular aspect still remains controversial and further studies should better clarify if the role of PRP in bonegraft integration is limited in favouring just the first healing phases.

Also with respect to the graft maturation process, the literature is not univocal, but in this case PRP seems to have a more positive influence. The studies led by Orrego et al., Radice et al., and Seijas et. al proved that PRP augmentation is able to determine a faster and better graft maturation with respect to the control groups $[23,44,53]$. In these trials MRI evaluations revealed that at 4 to 12 months after surgery the signal of the graft in the PRP group was significantly more homogenous and comparable to that of the intact posterior cruciate ligament. Radice et al. [23] reported that PRP augmentation contributed to reducing the time needed to have a homogenous, low-intensity graft signal by almost $50 \%$. Besides MRI findings, Sánchez et al. were able to perform arthroscopic and histological evaluations in a selected group of patients who underwent second-look arthroscopies [22]. The biopsies from PRP-augmented grafts confirmed the superior tissue quality that was hypothesized at imaging. Two studies did not show a significantly better graft maturation following PRP augmentation [50, 51]: however, in both these studies PRP determined superior MRI results and the lack of statistical significance might be attributed to the low sample size included in these trials [54]. Based on this evidence, it is possible to assume that PRP might positively influence graft maturation.

Finally, another important aspect regarding the clinical benefit that could be related to the observed tissue changes in terms of graft integration and maturation. The literature analysis on the clinical outcome of ACL reconstruction did not show any superior results with PRP augmentation. However, the majority of the published clinical studies did not consider clinical results as the primary outcome of the biological augmentation. Clinical evaluations were carried out in only a few studies and were limited to short-term followup, which prevents an accurate analysis of difference in failure rate and revision surgeries between the treatment groups, and further studies at longer followup should focus the real overall clinical benefit of PRP.

The present literature analysis is affected by some limitations. First of all each trial involves a different technique for ACL reconstruction: different types of graft were used, different tunneling, and also different fixation devices, so comparison of results is difficult and, at least hypothetically, it should be considered that some techniques might have a better response to biological stimulation than others. Furthermore, PRP has been administered in different ways: some authors applied it by a simple intra-articular injection after the procedure, others used it to cover the graft before or after its intra-articular fixation, and others injected it into the graft, and PRP could be even combined with substrates to increase ACL augmentation. Moreover, besides the intra-articular area of the graft, some authors also applied PRP inside the bone tunnels: some in both the tibial and femoral one and some only in the femoral one. Therefore, different applicative methods on different specific targets might determine different outcomes. Finally, another confounding factor is directly linked to PRP itself, that is, a well-known and debated topic, that is, the dramatic variability among different PRP formulations [55]. Currently there are several different PRPs in clinical use, differing in terms of preparation methods, activation, and cell content. These features affect the final concentration of GFs and other bioactive molecules delivered in situ and might influence the overall regenerative potential of PRP. In light of these aspects, it is even harder to summarize results of clinical trials: each author uses his own formulation and so it is impossible, at present, to establish the best PRP for improving ACL reconstruction, as well as the real potential of this biological approach for ACL augmentation. Considering the great interproduct variability and the various applicative strategies, further high quality studies are needed to determine the best formulation, administration modalities, and the best responding targets of PRP augmentation in ACL reconstructive surgery. 


\section{Conclusion}

This systematic review underlines that clinical results on PRP use for ACL augmentation are controversial. The intraoperative use of PRP proved to be safe, and PRP actually showed to even reduce the surgical morbidity promoting graft harvest site healing. Based on current evidence, PRP seems to play a positive role in the healing mechanisms after ACL surgery for what regards graft maturation, whereas the majority of the studies showed no benefit in terms of graft integration, especially in preventing bone tunnel widening. Finally, PRP did not provide a superior clinical outcome at short-term followup, whereas data at longer followup are lacking to address the overall clinical benefit of PRP augmentation.

\section{Conflict of Interests}

This work was partially supported by Italian Ministry of Health (Project "Ricerca Finalizzata"-2009-1498841).

\section{References}

[1] A. M. Buoncristiani, F. P. Tjoumakaris, J. S. Starman, M. Ferretti, and F. H. Fu, "Anatomic double-bundle anterior cruciate ligament reconstruction," Arthroscopy, vol. 22, no. 9, pp. 10001006, 2006.

[2] K. B. Freedman, M. J. D’Amato, D. D. Nedeff, A. Kaz, and B. R. Bach Jr., "Arthroscopic anterior cruciate ligament reconstruction: a metaanalysis comparing patellar tendon and hamstring tendon autografts," The American Journal of Sports Medicine, vol. 31, no. 1, pp. 2-11, 2003.

[3] H. S. Kim, J. K. Seon, and A. R. Jo, "Current trends in anterior cruciate ligament reconstruction," Knee Surgery \& Related Research, vol. 25, no. 4, pp. 165-173, 2013.

[4] S. Abe, M. Kurosaka, T. Iguchi, S. Yoshiya, and K. Hirohata, "Light and electron microscopic study of remodeling and maturation process in autogenous graft for anterior cruciate ligament reconstruction," Arthroscopy, vol. 9, no. 4, pp. 394-405, 1993.

[5] S. Cho, T. Muneta, S. Ito, K. Yagishita, and S. Ichinose, "Electron microscopic evaluation of two-bundle anatomically reconstructed anterior cruciate ligament graft," Journal of Orthopaedic Science, vol. 9, no. 3, pp. 296-301, 2004.

[6] R. P. Falconiero, V. J. DiStefano, and T. M. Cook, "Revascularization and ligamentization of autogenous anterior cruciate ligament grafts in humans," Arthroscopy, vol. 14, no. 2, pp. 197205, 1998.

[7] S. M. Howell, K. E. Knox, T. E. Farley, and M. A. Taylor, "Revascularization of a human anterior cruciate ligament graft during the first two years of implantation," The American Journal of Sports Medicine, vol. 23, no. 1, pp. 42-49, 1995.

[8] L. L. Johnson, "The outcome of a free autogenous semitendinosus tendon graft in human anterior cruciate reconstructive surgery: a histological study," Arthroscopy, vol. 9, no. 2, pp. 131142, 1993.

[9] C. Signorelli, T. Bonanzinga, N. Lopomo et al., "Do preoperative knee laxity values influence post-operative ones after anterior cruciate ligament reconstruction?" Scandinavian Journal of Medicine and Science in Sports, vol. 23, no. 4, pp. e219e224, 2013.
[10] B. Di Matteo, G. Filardo, E. Kon, and M. Marcacci, "Plateletrich plasma: evidence for the treatment of patellar and Achilles tendinopathy-a systematic review," MUSCULOSKELETAL SURGERY, vol. 99, no. 1, pp. 1-9, 2015.

[11] E. Kon, G. Filardo, B. Di Matteo, and M. Marcacci, "PRP for the treatment of cartilage pathology," The Open Orthopaedics Journal, vol. 7, pp. 120-128, 2013.

[12] T. Yuan, C. Q. Zhang, and J. H. Wang, "Augmenting tendon and ligament repair with platelet-rich plasma (PRP)," Muscle, Ligaments and Tendons Journal, vol. 3, no. 3, pp. 139-149, 2013.

[13] G. Filardo, E. Kon, A. Roffi, B. Di Matteo, M. L. Merli, and M. Marcacci, "Platelet-rich plasma: why intra-articular? A systematic review of preclinical studies and clinical evidence on PRP for joint degeneration," Knee Surgery, Sports Traumatology, Arthroscopy, 2013.

[14] N. A. Smyth, C. D. Murawski, L. A. Fortier, B. J. Cole, and J. G. Kennedy, "Platelet-rich plasma in the pathologic processes of cartilage: review of basic science evidence," Arthroscopy, vol. 29, no. 8, pp. 1399-1409, 2013.

[15] L. Engebretsen, K. Steffen, J. Alsousou et al., "IOC consensus paper on the use of platelet-rich plasma in sports medicine," British Journal of Sports Medicine, vol. 44, no. 15, pp. 1072-1081, 2010.

[16] N. Baksh, C. P. Hannon, C. D. Murawski, N. A. Smyth, and J. G. Kennedy, "Platelet-rich plasma in tendon models: a systematic review of basic science literature," Arthroscopy, vol. 29, no. 3, pp. 596-607, 2013.

[17] S. G. Boswell, B. J. Cole, E. A. Sundman, V. Karas, and L. A. Fortier, "Platelet-rich plasma: a milieu of bioactive factors," Arthroscopy, vol. 28, no. 3, pp. 429-439, 2012.

[18] A. M. Biercevicz, D. L. Miranda, J. T. MacHan, M. M. Murray, and B. C. Fleming, "In situ, noninvasive, $\mathrm{T}^{*}$-weighted mriderived parameters predict Ex vivo structural properties of an anterior cruciate ligament reconstruction or bioenhanced primary repair in a porcine model," The American Journal of Sports Medicine, vol. 41, no. 3, pp. 560-566, 2013.

[19] R. Kuroda, M. Kurosaka, S. Yoshiya, and K. Mizuno, "Localization of growth factors in the reconstructed anterior cruciate ligament: immunohistological study in dogs," Knee Surgery, Sports Traumatology, Arthroscopy, vol. 8, no. 2, pp. 120-126, 2000.

[20] M. Slater, J. Patava, K. Kingham, and R. S. Mason, "Involvement of platelets in stimulating osteogenic activity," Journal of Orthopaedic Research, vol. 13, no. 5, pp. 655-663, 1995.

[21] P. Vavken, F. A. Saad, and M. M. Murray, "Age dependence of expression of growth factor receptors in porcine ACL fibroblasts," Journal of Orthopaedic Research, vol. 28, no. 8, pp. 1107-1112, 2010.

[22] M. Sánchez, E. Anitua, J. Azofra, R. Prado, F. Muruzabal, and I. Andia, "Ligamentization of tendon grafts treated with an endogenous preparation rich in growth factors: gross morphology and histology," Arthroscopy, vol. 26, no. 4, pp. 470-480, 2010.

[23] F. Radice, R. Yánez, V. Gutiérrez, J. Rosales, M. Pinedo, and S. Coda, "Comparison of magnetic resonance imaging findings in anterior cruciate ligament grafts with and without autologous platelet-derived growth factors," Arthroscopy, vol. 26, no. 1, pp. 50-57, 2010.

[24] A. M. de Almeida, M. K. Demange, M. F. Sobrado, M. B. Rodrigues, A. Pedrinelli, and A. J. Hernandez, "Patellar tendon healing with platelet-rich plasma: a prospective randomized 
controlled trial," The American Journal of Sports Medicine, vol. 40, no. 6, pp. 1282-1288, 2012.

[25] M. Cervellin, L. de Girolamo, C. Bait, M. Denti, and P. Volpi, "Autologous platelet-rich plasma gel to reduce donorsite morbidity after patellar tendon graft harvesting for anterior cruciate ligament reconstruction: a randomized, controlled clinical study," Knee Surgery, Sports Traumatology, Arthroscopy, vol. 20, no. 1, pp. 114-120, 2012.

[26] X. Xie, S. Zhao, H. Wu et al., "Platelet-rich plasma enhances autograft revascularization and reinnervation in a dog model of anterior cruciate ligament reconstruction," Journal of Surgical Research, vol. 183, no. 1, pp. 214-222, 2013.

[27] X. Xie, H. Wu, S. Zhao, G. Xie, X. Huangfu, and J. Zhao, “The effect of platelet-rich plasma on patterns of gene expression in a dog model of anterior cruciate ligament reconstruction," Journal of Surgical Research, vol. 180, no. 1, pp. 80-88, 2013.

[28] A. N. Mastrangelo, P. Vavken, B. C. Fleming, S. L. Harrison, and M. M. Murray, "Reduced platelet concentration does not harm PRP effectiveness for ACL repair in a porcine in vivo model," Journal of Orthopaedic Research, vol. 29, no. 7, pp. 1002-1007, 2011.

[29] S. M. Joshi, A. N. Mastrangelo, E. M. Magarian, B. C. Fleming, and M. M. Murray, "Collagen-platelet composite enhances biomechanical and histologic healing of the porcine anterior cruciate ligament," The American Journal of Sports Medicine, vol. 37, no. 12, pp. 2401-2410, 2009.

[30] M. M. Murray, M. Palmer, E. Abreu, K. P. Spindler, D. Zurakowski, and B. C. Fleming, "Platelet-rich plasma alone is not sufficient to enhance suture repair of the ACL in skeletally immature animals: an in vivo study," Journal of Orthopaedic Research, vol. 27, no. 5, pp. 639-645, 2009.

[31] M. M. Murray, K. P. Spindler, E. Abreu et al., "Collagen-platelet rich plasma hydrogel enhances primary repair of the porcine anterior cruciate ligament," Journal of Orthopaedic Research, vol. 25, no. 1, pp. 81-91, 2007.

[32] M. M. Murray, K. P. Spindler, P. Ballard, T. P. Welch, D. Zurakowski, and L. B. Nanney, "Enhanced histologic repair in a central wound in the anterior cruciate ligament with a collagenplatelet-rich plasma scaffold," Journal of Orthopaedic Research, vol. 25, no. 8, pp. 1007-1017, 2007.

[33] M. M. Murray, K. P. Spindler, C. Devin et al., "Use of a collagenplatelet rich plasma scaffold to stimulate healing of a central defect in the canine ACL," Journal of Orthopaedic Research, vol. 24, no. 4, pp. 820-830, 2006.

[34] A. Weiler, C. Förster, P. Hunt et al., “The influence of locally applied platelet-derived growth factor-BB on free tendon graft remodeling after anterior cruciate ligament reconstruction," The American Journal of Sports Medicine, vol. 32, no. 4, pp. 881-891, 2004.

[35] R. Yoshida, M. Cheng, and M. M. Murray, "Increasing platelet concentration in platelet-rich plasma inhibits anterior cruciate ligament cell function in three-dimensional culture," Journal of Orthopaedic Research, vol. 32, no. 2, pp. 291-295, 2014.

[36] R. Yoshida and M. M. Murray, "Peripheral blood mononuclear cells enhance the anabolic effects of platelet-rich plasma on anterior cruciate ligament fibroblasts," Journal of Orthopaedic Research, vol. 31, no. 1, pp. 29-34, 2013.

[37] M. Cheng, V. M. Johnson, and M. M. Murray, "Effects of age and platelet-rich plasma on ACL cell viability and collagen gene expression," Journal of Orthopaedic Research, vol. 30, no. 1, pp. 79-85, 2012.
[38] L. Fallouh, K. Nakagawa, T. Sasho et al., "Effects of autologous platelet-rich plasma on cell viability and collagen synthesis in injured human anterior cruciate ligament," Journal of Bone and Joint Surgery, vol. 92, no. 18, pp. 2909-2916, 2010.

[39] E. M. Magarian, P. Vavken, and M. M. Murray, "Human anterior cruciate ligament fibroblasts from immature patients have a stronger in vitro response to platelet concentrates than those from mature individuals," Knee, vol. 18, no. 4, pp. 247-251, 2011.

[40] M. Cheng, H. Wang, R. Yoshida, and M. M. Murray, "Platelets and plasma proteins are both required to stimulate collagen gene expression by anterior cruciate ligament cells in threedimensional culture," Tissue Engineering A, vol. 16, no. 5, pp. 1479-1489, 2010.

[41] A. N. Mastrangelo, E. M. Magarian, M. P. Palmer, P. Vavken, and M. M. Murray, "The effect of skeletal maturity on the regenerative function of intrinsic ACL cells," Journal of Orthopaedic Research, vol. 28, no. 5, pp. 644-651, 2010.

[42] S. C. Scherping Jr., C. C. Schmidt, H. I. Georgescu, C. K. Kwoh, C. H. Evans, and S. L. Woo, "Effect of growth factors on the proliferation of ligament fibroblasts from skeletally mature rabbits," Connective Tissue Research, vol. 36, no. 1, pp. 1-8, 1997.

[43] M. Rupreht, M. Vogrin, and M. Hussein, "MRI evaluation of tibial tunnel wall cortical bone formation after platelet-rich plasma applied during anterior cruciate ligament reconstruction," Radiology and Oncology, vol. 47, no. 2, pp. 119-124, 2013.

[44] R. Seijas, O. Ares, J. Catala, P. Alvarez-Diaz, X. Cusco, and R. Cugat, "Magnetic resonance imaging evaluation of patellar tendon graft remodelling after anterior cruciate ligament reconstruction with or without platelet-rich plasma," Journal of Orthopaedic Surgery, vol. 21, no. 1, pp. 10-14, 2013.

[45] F. Mirzatolooei, M. T. Alamdari, and H. R. Khalkhali, “The impact of platelet-rich plasma on the prevention of tunnel widening in anterior cruciate ligament reconstruction using quadrupled autologous hamstring tendon: a randomised clinical trial," Journal of Bone and Joint Surgery, vol. 95-B, no. 1, pp. 65-69, 2013.

[46] R. A. Magnussen, D. C. Flanigan, A. D. Pedroza, K. A. Heinlein, and C. C. Kaeding, "Platelet rich plasma use in allograft ACL reconstructions: two-year clinical results of a MOON cohort study," Knee, vol. 20, no. 4, pp. 277-280, 2013.

[47] M. Rupreht, V. Jevtič, I. Serša, M. Vogrin, and M. Jevšek, "Evaluation of the tibial tunnel after intraoperatively administered platelet-rich plasma gel during anterior cruciate ligament reconstruction using diffusion weighted and dynamic contrastenhanced MRI," Journal of Magnetic Resonance Imaging, vol. 37, no. 4, pp. 928-935, 2013.

[48] A. Vadalà, R. Iorio, A. De Carli et al., "Platelet-rich plasma: does it help reduce tunnel widening after ACL reconstruction?" Knee Surgery, Sports Traumatology, Arthroscopy, vol. 21, no. 4, pp. 824-829, 2013.

[49] M. Vogrin, M. Rupreht, D. Dinevski et al., "Effects of a platelet gel on early graft revascularization after anterior cruciate ligament reconstruction: a prospective, randomized, doubleblind, clinical trial," European Surgical Research, vol. 45, no. 2, pp. 77-85, 2010.

[50] D. Figueroa, P. Melean, R. Calvo et al., "Magnetic resonance imaging evaluation of the integration and maturation of semitendinosus-gracilis graft in anterior cruciate ligament reconstruction using autologous platelet concentrate," Arthroscopy, vol. 26, no. 10, pp. 1318-1325, 2010. 
[51] J. R. Valentí Nin, G. Mora Gasque, A. Valentí Azcárate, J. D. Aquerreta Beola, and M. Hernandez Gonzalez, "Has plateletrich plasma any role in anterior cruciate ligament allograft healing?" Arthroscopy, vol. 25, no. 11, pp. 1206-1213, 2009.

[52] A. Silva and R. Sampaio, "Anatomic ACL reconstruction: does the platelet-rich plasma accelerate tendon healing?" Knee Surgery, Sports Traumatology, Arthroscopy, vol. 17, no. 6, pp. 676-682, 2009.

[53] M. Orrego, C. Larrain, J. Rosales et al., "Effects of platelet concentrate and a bone plug on the healing of hamstring tendons in a bone tunnel," Arthroscopy, vol. 24, no. 12, pp. 13731380, 2008.

[54] P. Vavken, P. Sadoghi, and M. M. Murray, "The effect of platelet concentrates on graft maturation and graft-bone interface healing in anterior cruciate ligament reconstruction in human patients: a systematic review of controlled trials," Arthroscopy, vol. 27, no. 11, pp. 1573-1583, 2011.

[55] M. Tschon, M. Fini, R. Giardino et al., "Lights and shadows concerning platelet products for musculoskeletal regeneration," Frontiers in Bioscience, vol. 3, no. 1, pp. 96-107, 2011. 


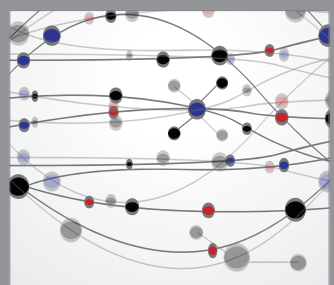

The Scientific World Journal
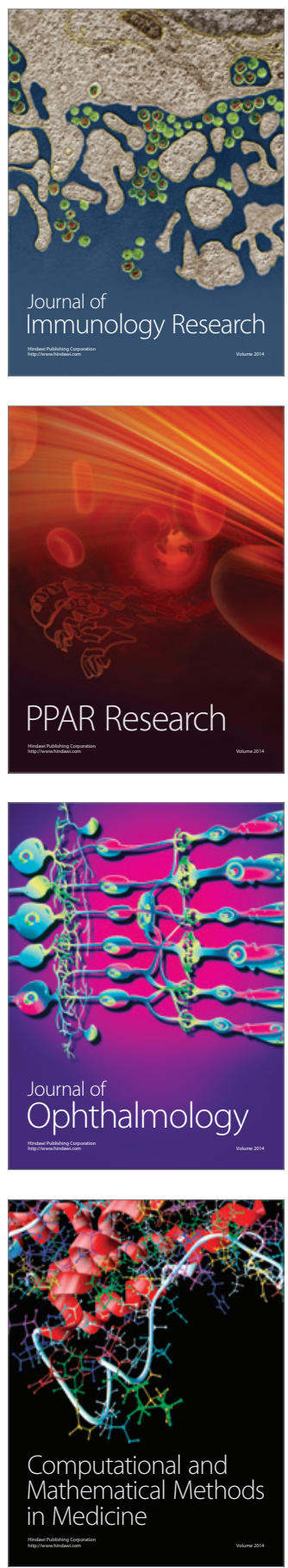

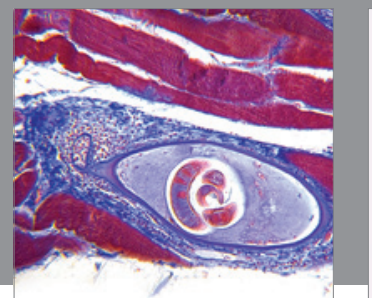

Gastroenterology

Research and Practice
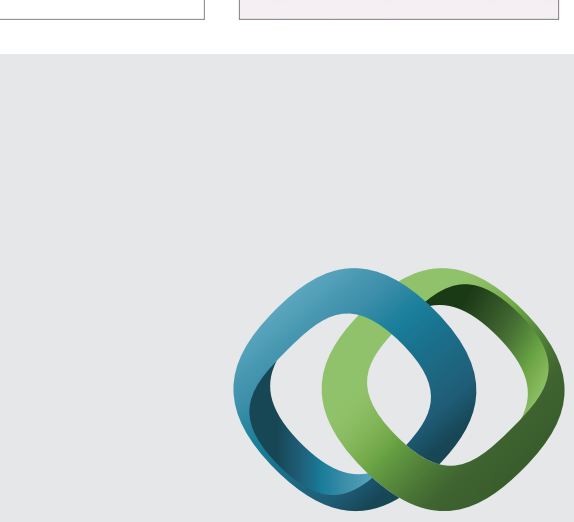

\section{Hindawi}

Submit your manuscripts at

http://www.hindawi.com
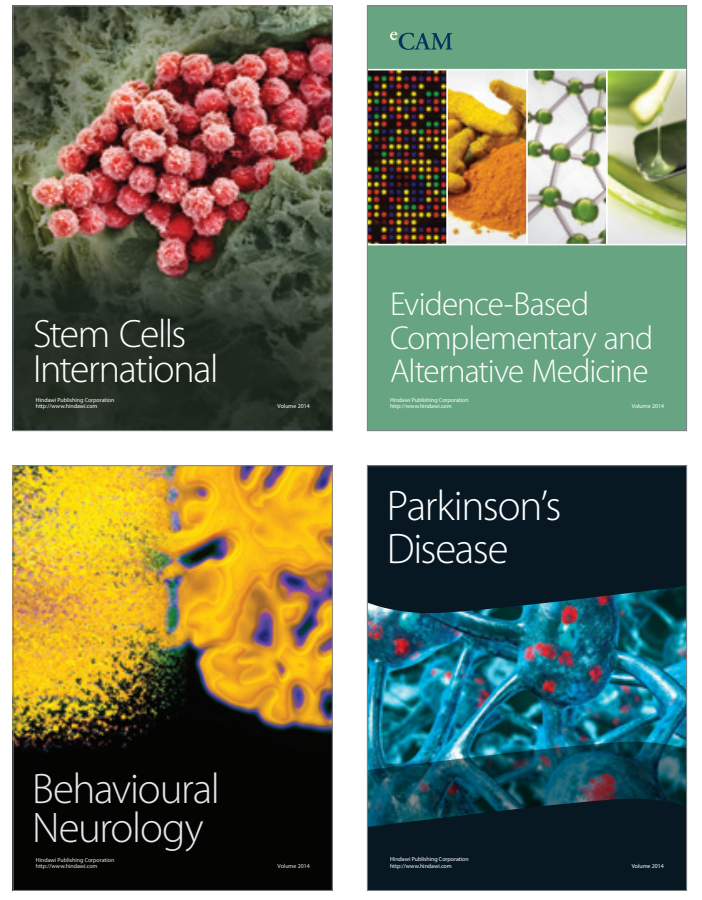
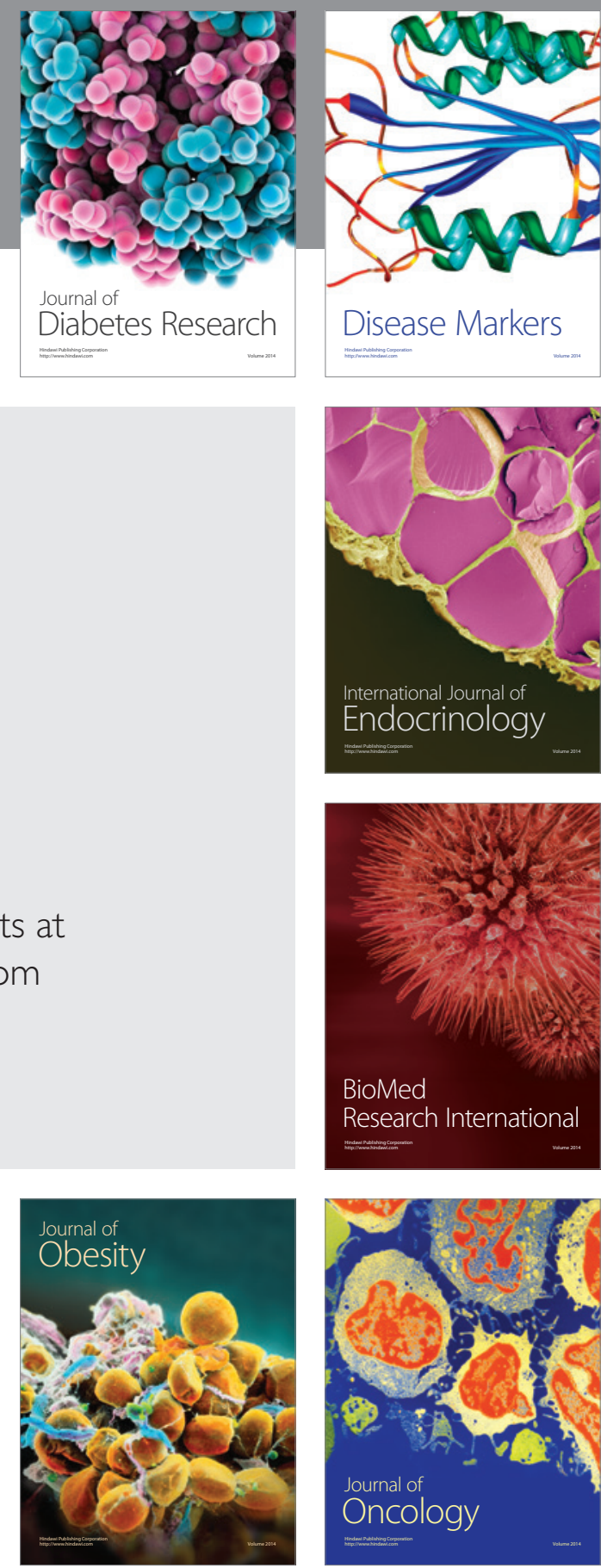

Disease Markers
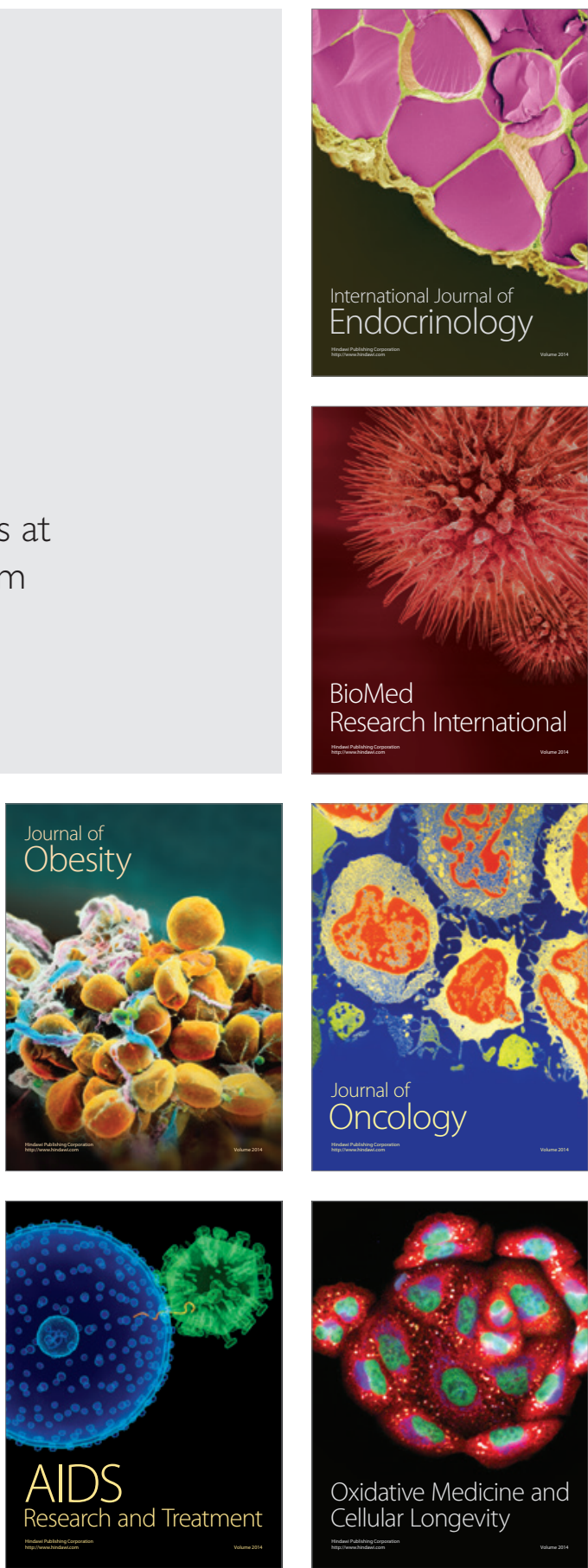